\title{
Phosphate Availability Modulates Root Exudate Composition and Rhizosphere Microbial Community in a Teosinte and a Modern Maize Cultivar
}

\author{
Vanessa L. Brisson, ${ }^{1,2, \dagger}$ Jesper Richardy, ${ }^{3}$ Suzanne M. Kosina, ${ }^{1}$ Trent R. Northen, ${ }^{1,4}$ John P. Vogel, ${ }^{4,5}$ and Amélie C. M. Gaudin ${ }^{3}$ \\ ${ }^{1}$ Environmental Genomics and Systems Biology, Lawrence Berkeley National Laboratory, Berkeley, CA 94720 \\ ${ }^{2}$ Biosciences and Biotechnology Division, Physical and Life Sciences Directorate, Lawrence Livermore National Laboratory, Livermore, CA 94550 \\ ${ }^{3}$ Department of Plant Sciences, University of California at Davis, Davis, CA 95616 \\ ${ }^{4}$ The DOE Joint Genome Institute, Lawrence Berkeley National Laboratory, Berkeley, CA 94720 \\ ${ }^{5}$ Department of Plant and Microbial Biology, University of California, Berkeley, CA 94720
}

Accepted for publication 30 June 2021.

\begin{tabular}{|c|c|}
\hline $\begin{array}{l}\text { Domestication and breeding have affected interactions } \\
\text { between plants and their microbiomes in ways that are only } \\
\text { beginning to be understood but may have important implications } \\
\text { for recruitment of rhizosphere microorganisms, particularly } \\
\text { under stress conditions. We investigated the responses of a } \\
\text { modern maize (Zea mays subsp. mays) cultivar and its wild } \\
\text { relative, teosinte (Z. mays subsp. parviglumis), to different } \\
\text { phosphate availabilities. We appraised responses of the plant- } \\
\text { microbial holobiont to phosphate stresses by profiling root exu- } \\
\text { date metabolomes, and microbial communities in the root endo- } \\
\text { sphere and rhizosphere. We also performed plate assays to } \\
\text { quantify phosphate-solubilizing microorganisms from the rhizo- } \\
\text { sphere. Although root exudate metabolite profiles were distinct } \\
\text { between the teosinte and modern maize under high phosphate, } \\
\text { both plants shifted exudate compositions in response to phos- } \\
\text { phate stress toward a common metabolite profile. Root and rhi- }\end{array}$ & $\begin{array}{l}\text { zosphere microbial communities also responded significantly to } \\
\text { both plant type and the phosphate availability. A subset of bac- } \\
\text { terial and fungal taxa were differentially abundant under the dif- } \\
\text { ferent phosphate conditions, with each of the three conditions } \\
\text { favoring different taxa. Both teosinte and maize rhizospheres } \\
\text { harbored phosphate-solubilizing microorganisms under all } \\
\text { growth conditions. These results suggest that the root exudation } \\
\text { response to phosphate stress was conserved through the } \\
\text { domestication of maize from teosinte, shifting exudation levels } \\
\text { of specific metabolites. Although microbial communities also } \\
\text { shifted, plate-based assays did not detect selective recruitment } \\
\text { of phosphate solubilizers in response to phosphate availability. } \\
\text { Keywords: maize, metabolomics, microbiome, phosphate- } \\
\text { solubilizing microorganisms, phosphate stress, rhizosphere and } \\
\text { phyllosphere, root exudates, teosinte, Zea mays }\end{array}$ \\
\hline
\end{tabular}

${ }^{\dagger}$ Corresponding author: V. L. Brisson; vbrisson@berkeley.edu

Funding: This work was supported by the Laboratory Directed Research and Development Program of Lawrence Berkeley National Laboratory with use of the United States Department of Energy (DOE) Office of Science User Facility resources at the DOE Joint Genome Institute and at the DOE National Energy Research Scientific Computing Center under DOE contract number DE-AC02-05CH11231. This work was performed under the auspices of the DOE by Lawrence Livermore National Laboratory under Contract DE-AC52-07NA27344. LLNL IM release number LLNL-JRNL-817896. This work was also partially funded by the Foundation for Food and Agriculture Research, the United States Department of Agriculture-National Institute of Food and Agriculture, Agricultural Experimental Station Project CA-D-PLS-2332-H to A. C. M. Gaudin.

*The $\boldsymbol{e}$-Xtra logo stands for "electronic extra" and indicates that supplementary figures and supplementary tables are published online.

The author(s) declare no conflict of interest.

This article is in the public domain and not copyrightable. It may be freely reprinted with customary crediting of the source. The American Phytopathological Society, 2022.
The domestication of maize from the wild grass teosinte began over 7,000 years ago (Ranum et al. 2014). Although maize has primarily been bred for desirable aboveground traits, including apical dominance and seed size, belowground traits such as root morphology and rhizosphere microbial community structure have been affected by the genetic changes acquired through breeding (Brisson et al. 2019; Doebley et al. 1997; Pérez-Jaramillo et al. 2016; Schmidt et al. 2016, 2020a). Studies of rhizosphere communities from different inbred maize genotypes suggest that host plant genotypes are strong drivers of microbial community composition, along

Author contributions: V.B., J.V., T.R., and A.G. designed the study. V.B. and J.R. conducted the experiments. V.B. collected the samples and performed metabolite and DNA extractions. S.K. conducted the LCMS/MS metabolomics analysis. V.B. and S.K. analyzed the LCMS/MS data. V.B. analyzed the sequencing data. All authors contributed to the drafting of this manuscript. 
with other factors such as soil fertility and growth stage (Peiffer et al. 2013; Walters et al. 2018). Rhizosphere microbial communities of teosinte and maize have also been shown to differ (Brisson et al. 2019; Schmidt et al. 2020b; Szoboszlay et al. 2015) but changes in microbial community recruitment do not follow a clear progression along evolutionary gradients, suggesting complex effects of selective pressure on plant-driven microbiome recruitment (Brisson et al. 2019; Emmett et al. 2018; Schmidt et al. 2020b). The changes in belowground interactions and their implications for plant nutrition are only beginning to be understood (Brisson et al. 2019; Schmidt et al. 2020a, b) and improved mechanistic understanding of rhizosphere processes and their response to selective pressures could contribute to rhizosphere engineering for sustainable agriculture.

Plant-associated microbial communities are critical for supporting plant growth and can confer fitness and productivity advantages through several direct and indirect mechanisms that promote growth, nutrient acquisition, stress tolerance, and resistance to pathogens (Trivedi et al. 2020). Of particular importance are some rhizosphere microorganisms that can help plants acquire scarce or nonbioavailable nutrients, including phosphorus (P), nitrogen, iron, and sulfur (Jacoby et al. 2017; Pii et al. 2015). Improving P availability, either through phosphate solubilization from phosphate minerals or through mineralization of organic phosphates, has been studied as an important mechanism of plant growth promotion by microorganisms (Alori et al. 2017).

Phosphate-solubilizing microorganisms are important members of soil microbial communities that can release otherwise insoluble phosphate from minerals, making it available for plant growth (Alori et al. 2017). Phosphate solubilizers include both bacteria and fungi from a wide range of species which can solubilize phosphate from minerals through acidification, complexation of associated anions, or exchange reactions (Alori et al. 2017; Arcand and Schneider 2006). Organic acids from both plants and microorganisms can contribute to phosphate solubilization through all of these mechanisms and are thought to be the main mechanism by which phosphate-solubilizing microorganisms release phosphate (Brisson et al. 2016; Rodríguez and Fraga 1999).

The composition of root exudates is a main mechanism for selective recruitment of microorganisms to the rhizosphere and adaptation to abiotic stress conditions (Trivedi et al. 2020). In maize, the composition of root exudates differs between plant genotypes and in response to changes in the availability of nitrogen, $\mathrm{P}$, potassium, and iron (Carvalhais et al. 2011; Cheng et al. 2018). In turn, root exudates have been shown to shape rhizosphere microbial communities by favoring some microorganisms over others (Huang et al. 2019; Zhalnina et al. 2018). Root exudates also contain organic acids that may directly contribute to phosphate solubilization (Edayilam et al. 2018; Hinsinger 2001).

The ability of plants to recruit potentially beneficial microorganism under stress conditions may have been modified by extensive breeding under conditions of high nutrient inputs in modern agricultural systems. The increased availability of nutrients may have decoupled plant productivity from the need to maintain nutrient-acquiring capabilities via rhizosphere microbial communities (Porter and Sachs 2020). Compared with inbred maize and modern hybrids, teosinte and landraces were more able to take advantage of nutrients from organically managed soil to increase growth (Schmidt et al. 2020b); however, mechanistic impacts on phosphate acquisition remain unknown. Because heterosis for maize root traits related to higher phosphate acquisition are only found under low-phosphate conditions (Liu et al. 2018), phosphate efficiency in maize is likely not under strong selection when breeding for yield, potentially compromising beneficial association useful for modern maize production under low-input scenarios. Without selective pressure, the ability to recruit beneficial microbiomes may have been affected. A study addressing the relationship between phosphate stress response and root microbial community in Arabidopsis thaliana found that mutations in known phosphate stress-response genes in the plant were associated with differential recruitment of bacterial taxa (Castrillo et al. 2017).

In this study, we investigated the influence of phosphate availability on root exudation, and on rhizosphere and root microbial community assembly in a teosinte and a modern maize cultivar. We hypothesized that the root exudate compositions of teosinte and modern maize would differ but would both change in response to phosphate availability, with increased or decreased exudation of specific metabolites regulating phosphate availability or interactions with microorganisms. We also hypothesized that genotypes would recruit different rhizosphere microbiomes, and preferentially recruit phosphate-solubilizing microorganisms to the rhizosphere when grown on an insoluble phosphate source. We expected that teosinte might be more effective at preferential recruitment of phosphate-solubilizing microorganisms because modern maize has not been subjected to extensive selection under conditions of high nutrient availability and may be less able to adapt to low or insoluble phosphate.

\section{MATERIALS AND METHODS}

Plants and growth conditions. One teosinte accession (Zea mays subsp. parviglumis Pi566688) and one modern maize accession (Z. mays subsp. mays hybrid 3489) were used in this work. Teosinte seed were obtained from the National Plant Germplasm System. Modern maize seed were obtained from Dupont Pioneer. Seed were surface sterilized by soaking in a solution of $10 \%$ bleach with $0.01 \%$ Triton-X for $8 \mathrm{~min}$. Sterilized seed were rinsed six times with sterile water, then germinated in Petri dishes on autoclaved filter paper soaked with sterile water. After 2 days, germinated seed were transferred to prepared pots at approximately $1.5 \mathrm{~cm}$ of depth and subjected to three phosphate treatments: high soluble phosphate, low soluble phosphate, and insoluble phosphate (Table 1).

One-gallon (3.78 liters) pots were filled with autoclaved sand to control nutrient supply and microbial activity, and phosphate was added according to treatment levels detailed in Table 1. For the insoluble phosphate condition, $2.7 \mathrm{mmol}(0.5 \mathrm{~g})$ of $\mathrm{FePO}_{4} \cdot 2 \mathrm{H}_{2} \mathrm{O}$ was mixed into the sand $(5 \mathrm{~cm}$ across and $3 \mathrm{~cm}$ deep) in the middle of the pot where the seedling was to be planted. Media were prepared

TABLE 1

Growth conditions

\begin{tabular}{llll}
\hline Condition & $\begin{array}{c}\mathrm{FePO}_{4} \cdot 2 \mathrm{H}_{2} \mathrm{O} \\
\text { added to pot }\end{array}$ & $\begin{array}{c}\mathrm{KH}_{2} \mathrm{PO}_{4} \text { concentration } \\
\text { in medium }\end{array}$ & $\begin{array}{c}\text { Total } \mathrm{PO}_{4}{ }^{3-} \text { provided } \\
\text { over experiment (mmol) }\end{array}$ \\
\hline High soluble phosphate & $0 \mathrm{mmol}(0 \mathrm{~g})$ & $1.25 \mathrm{mM}(0.17 \mathrm{~g} /$ liter $)$ & 2.4 \\
\hline Low soluble phosphate & $0 \mathrm{mmol}(0 \mathrm{~g})$ & $0.125 \mathrm{mM}(0.017 \mathrm{~g} /$ liter $)$ & 0.24 \\
\hline Insoluble phosphate & $2.7 \mathrm{mmol}(0.5 \mathrm{~g})$ & $0 \mathrm{mM}(0 \mathrm{~g} /$ liter $)$ & 2.7 \\
\hline
\end{tabular}


from Murashige and Skoog medium (Murashige and Skoog 1962) without phosphate (Caisson Labs, Smithfield, UT, U.S.A.) according to the manufacturer's instructions, with added $\mathrm{KH}_{2} \mathrm{PO}_{4}$ for the soluble phosphate conditions. Each pot was saturated with 1 liter of the appropriate medium for the desired growth condition (Table 1). Ten replicate pots were prepared for each combination of plant accession (teosinte, modern maize, and unplanted control) and growth condition (high phosphate, low phosphate, and insoluble phosphate), and arranged in a completely randomized design on a greenhouse bench.

The high phosphate concentration was selected based on the concentration in full Murashige and Skoog medium. As a reference point, for a maximum recommended fertilization level for maize of $\mathrm{P}_{2} \mathrm{O}_{4}$ at $80 \mathrm{lb} . / \mathrm{acre}(89.7 \mathrm{~kg} / \mathrm{ha})$ (Shapiro et al. 2008) and typical plant density of 30,000 plants/acre (74,000 plants/ha) (USDA-NASS 2021), fertilization in practice can consist of $\mathrm{P}$ at up to $8.5 \mathrm{mmol} / \mathrm{maize}$ plant. In our experiment, we provided $\mathrm{P}$ at a total of $2.4 \mathrm{mmol} / \mathrm{plant}$ over the course of 3 weeks of growth in the high phosphate condition. The low phosphate concentration was selected based on preliminary experiments in which this concentration was determined to produce visible phosphate stress within the 3-week timeframe of the experiment.

Immediately following planting, seedlings were inoculated with a microbial community soil suspension. Inoculum was prepared by suspending $180 \mathrm{~g}$ soil in 1 liter of Murashige and Skoog medium without added phosphate and shaking vigorously for $1 \mathrm{~min}$. Soil was sourced from the Russell Ranch Sustainable Agriculture Facility at the University of California-Davis from a rainfed, unfertilized wheat plot (Brisson et al. 2019). Each seedling was inoculated with $10 \mathrm{ml}$ of the inoculum. Seedlings were reinoculated with $10 \mathrm{ml}$ of freshly prepared inoculum 7 days after planting. Pots were fertilized weekly with $300 \mathrm{ml}$ of the appropriate medium. Between fertilizations, plants were watered twice weekly with $150 \mathrm{ml}$ of deionized water, which was sufficient to saturate the sand. Plants were grown for 21 days without supplemental lighting or heating (temperature range 16 to $32^{\circ} \mathrm{C}$ ). The duration of the experiment was chosen to be long enough to observe phosphate limitation phenotype in leaves but short enough to prevent pots from becoming rootbound.

Sample collection. After 3 weeks of growth, root exudates, plants, and soil samples were collected for analysis of plant growth, root exudate metabolomics, microbial communities, and microbial phosphate solubilization assays. Plants were carefully removed from the soil (sand) and gently shaken to remove bulk soil from the roots. Plant roots were rinsed in $150 \mathrm{ml}$ of $0.9 \%(154 \mathrm{mM}) \mathrm{NaCl}$ in distilled water to collect rhizosphere soil (Barillot et al. 2013). Excess $\mathrm{NaCl}$ solution was removed by decanting, and soil samples were frozen for further analysis. Soil from the unplanted control pots was harvested by collecting $20 \mathrm{ml}$ of soil from the center of the pots and stored frozen for further analysis.

Root exudate collection was conducted under the same greenhouse conditions as growth. Rinsed roots, with shoots still attached, were placed in $250 \mathrm{ml}$ of distilled water to equilibrate for $1 \mathrm{~h}$ prior to exudate collection. Plants were then transferred to $250 \mathrm{ml}$ of fresh distilled water, and root exudates were collected for $1.5 \mathrm{~h}$ (Zhalnina et al. 2018). Exudates were filtered with $0.22-\mu \mathrm{m}$ polyethersulfone filters (Corning, Tewksbury, MA, U.S.A.) and frozen until metabolite extraction. After exudate collection, root samples were collected for microbial community analysis. Approximately $3 \mathrm{~cm}$ of root was collected from the tip of the longest root and flash frozen for analysis.

Biomass and shoot elemental analysis. Plants were then separated into roots and shoots, and tissues were dried at $28^{\circ} \mathrm{C}$ for 1 week, at which point dry biomass weight was measured. Dried shoots were then ground to a powder and analyzed for elemental composition, including P content, using the acid digest method (Huang and Schulte 1985) at the Pennsylvania State University Agricultural Analytical Services Laboratory (University Park, PA, U.S.A.).
Metabolomics analysis. Filtered root exudate $(30 \mathrm{ml})$ was lyophilized, and metabolites were extracted in $1 \mathrm{ml}$ of highperformance liquid chromatography-grade methanol. The suspension was vortexed and incubated in a sonicating water bath with ice for $30 \mathrm{~min}$. Samples were then dried in a vacuum centrifuge to complete dryness and resuspended in $150 \mu$ of $0.0005 \times{ }^{13} \mathrm{C},{ }^{15} \mathrm{~N}$ amino acid mixture in methanol (Sigma-Aldrich, 767964). The solution was then vortexed, sonicated, centrifuged as described above, and filter sterilized through a $0.2-\mu \mathrm{m}$ pore size filter.

Polar metabolites from extracts were chromatographically separated using hydrophilic interaction liquid chromatography and detected by high-resolution mass spectrometry. Analyses were performed using an InfinityLab Poroshell 120 HILIC-Z column on an Agilent 1290 stack connected to a Thermo Q-Exactive Hybrid Quadrupole-Orbitrap Mass Spectrometer equipped with a Heated Electrospray Ionization (HESI-II) source probe under the parameters specified in Supplementary Table S1. Briefly, a gradient elution was performed with collection of MS1 and MS2 mass spectra; a library of analytical standards analyzed under the same conditions was used to perform a targeted query of the sample files for matching $\mathrm{m} / \mathrm{z}$, retention time, and fragmentation spectra using Metabolite Atlas (https:// github.com/biorack/metatlas) (Bowen and Northen 2010; Yao et al. 2015). Metabolomics data were deposited at the Joint Genome Institute Joint Genome Portal under project ID FD 1280336, with raw data under SP 1280337 and analysis output under AP 1280338.

Microbial community analysis. DNA was extracted from bulk and rhizosphere soils and from roots for microbial community analysis. Approximately $100 \mathrm{mg}$ of each sample was placed in a 2-ml microcentrifuge tube with an autoclave sterilized steel ball, frozen in liquid nitrogen, and ground to a powder using a TissueLyser (Qiagen, Hilden, Germany). Ground samples were extracted using PlantDNAzol (Thermo Fisher Scientific, Waltham, MA, U.S.A.) according to the manufacturer's instructions. Extracted DNA was sequenced at the Argonne National Laboratory Environmental Sample Preparation and Sequencing Facility. The prokaryotic 16S-V4 region was sequenced using the 515F-Y (GTGYCAGCMGCCGC GGTAA) (Parada et al. 2016) and 806R (GGACTACNVGG GTWTCTAAT) (Apprill et al. 2015) primers, using PNA blockers to limit amplification of plant chloroplast DNA. Fungal internal transcribed spacer 1 (ITS1) was sequenced using the ITS1F (CTTGGTCATTTAGAGGAAGTAA) (Gardes and Bruns 1993) and ITS2 (GCTGCGTTCTTCATCGATGC) (White et al. 1990) primers. Raw sequencing read data were deposited in the NCBI Sequence Read Archive under the BioProject number PRJNA 686132 . Sequences were processed using DADA2 (Callahan et al. 2016), as previously described (Brisson et al. 2019). Relative abundances of amplicon sequence variants (ASVs) detected in at least four samples were included in subsequent analyses.

Phosphate-solubilizing microorganism assay. Plate-based assays were conducted to quantify the proportion of rhizosphere microorganisms capable of utilizing insoluble $\mathrm{FePO}_{4} \cdot 2 \mathrm{H}_{2} \mathrm{O}$ for growth. Plates were prepared from modified Pikovskaya (PVK) agar without yeast extract (Nautiyal 1999; Pikovskaya 1948). This modification removes $\mathrm{P}$ present in the yeast extract as an alternate $\mathrm{P}$ source and has been used in previous studies isolating and characterizing phosphate-solubilizing microorganisms (Brisson et al. 2016; Nautiyal 1999). Microbial suspensions were prepared from rhizosphere and corresponding bulk control soil samples by suspending $0.5 \mathrm{ml}$ of soil with $10 \mathrm{ml}$ of sterile salt solution $(\mathrm{NaCl}$ at $9 \mathrm{~g} /$ liter) and vortexing for $1 \mathrm{~min}$ to release cells. For each rhizosphere or bulk control soil sample, a $50-\mu \mathrm{l}$ aliquot was plated on modified PVK agar prepared with $\mathrm{KH}_{2} \mathrm{PO}_{4}$ at $2.2 \mathrm{~g} /$ liter. Another $50-\mu \mathrm{l}$ aliquot was plated on modified $\mathrm{PVK}$ agar prepared with $\mathrm{FePO}_{4} \cdot 2 \mathrm{H}_{2} \mathrm{O}$ at $3 \mathrm{~g} /$ liter. After 6 days of incubation at $28^{\circ} \mathrm{C}$, colony 
counts were performed on all plates. For each rhizosphere or control sample, the proportion of phosphate solubilizers was determined as the ratio of the number of colonies on the $\mathrm{FePO}_{4} \cdot 2 \mathrm{H}_{2} \mathrm{O}$ plates to the number of colonies on the $\mathrm{KH}_{2} \mathrm{PO}_{4}$ plates.

Statistical analysis. All statistical analyses were carried out in $\mathrm{R}$ 3.6.2. Differentially abundant metabolites were detected using analysis of variance (ANOVA). Impacts of plant accession, phosphate treatments, and latitudinal position in the greenhouse (which corresponded to a light gradient) on plant traits, phosphate solubilization, and differential abundance of metabolites were detected using linear mixed-effects models and ANOVA. Longitudinal position was also considered but did not show a significant effect and, therefore, was removed from the final models. A two-way ANOVA test was performed for each response variable and detected metabolite to determine whether plant accession, phosphate condition, or an interaction between them were significant fixed factors, considering replicates as random effects. Variation in light levels was observed qualitatively within the greenhouse during the experiment, with the south side receiving more light than the north side, and greenhouse placement effect was added as a covariate in the mixed model. Raw $P$ values for metabolite ANOVA tests were adjusted for multiple comparisons using the Benjamini-Hochberg adjustment. An effect was considered to be significant if the adjusted $P$ value was $<0.05$. Microbial community $16 \mathrm{~S}$ and ITS amplicon sequencing data analyses were performed using the R packages phyloseq ( $\alpha$ diversity and principal coordinate analysis [PCoA]) (McMurdie and Holmes 2013), indicspecies (indicator species analysis) (De Cáceres and Legendre 2009), and DESeq2 (differential abundance analysis) (Love et al. 2014). For PCoA and correlation analyses described below, ASV abundance data were transformed using the centered log ratio (CLR) (Aitchison 1982) in order to correct for the inherent compositionality of the data (Gloor et al. 2017).

Correlations between microbial community composition and other observed variables (root exudate profiles, biomass, and shoot $\mathrm{P}$ content) were assessed using the Mantel test to compare distance matrices generated from CLR-transformed ASV relative abundances to distance matrices generated from the other data (normalized root exudate metabolite profiles, total biomass, and shoot $\mathrm{P}$ content). Mantel tests were run for the bacterial and fungal communities separately in both the root and rhizosphere compartments using the "mantel" function in the "vegan" package in R with 9,999 permutations. Correlations between individual ASVs and individual root exudate metabolites or biomass properties were assessed based on Spearman correlations and using the Benjamini-Hochberg correction to account for multiple comparisons. Correlations were considered significant if the adjusted $P$ value was $<0.05$.

\section{RESULTS}

Phosphate stress differential affects shoot and root growth in a teosinte and modern maize. Plant accession and phosphate availability both significantly influenced plant biomass $(P<0.001)$ but both plant accessions responded similarly to shifts in phosphate availability, as shown by nonsignificant interaction terms (Supplementary Table S2). High soluble phosphate availability yielded greater total biomass than insoluble phosphate source for both the modern maize and the teosinte. Low phosphate conditions yielded lower total biomass than high or insoluble phosphate for teosinte but was not significantly different from either condition in modern maize. Total biomass for the modern maize was greater than that of

\section{A Plant Biomass}

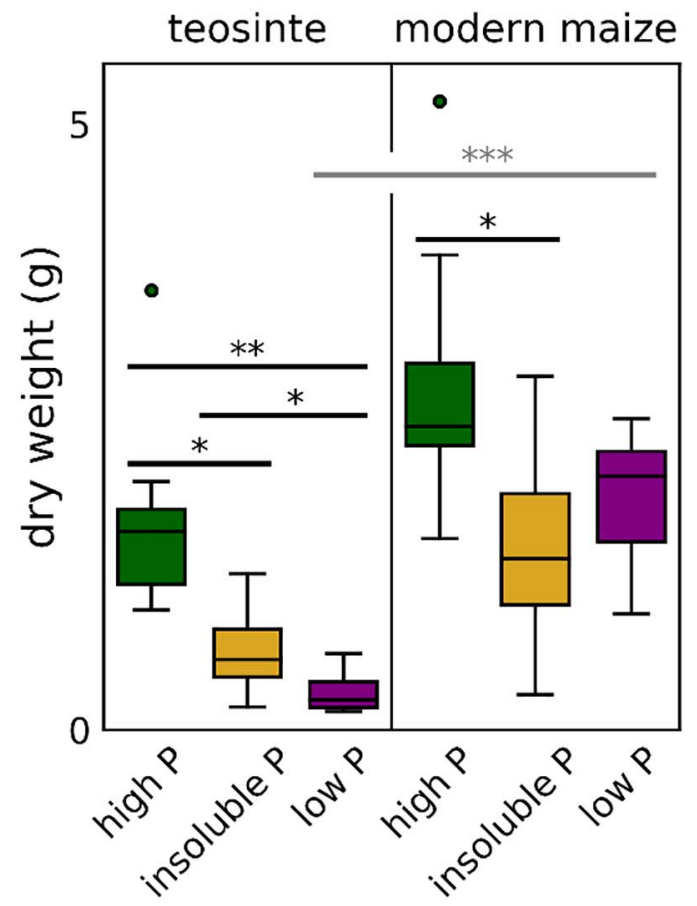

B Root Biomass Proportion

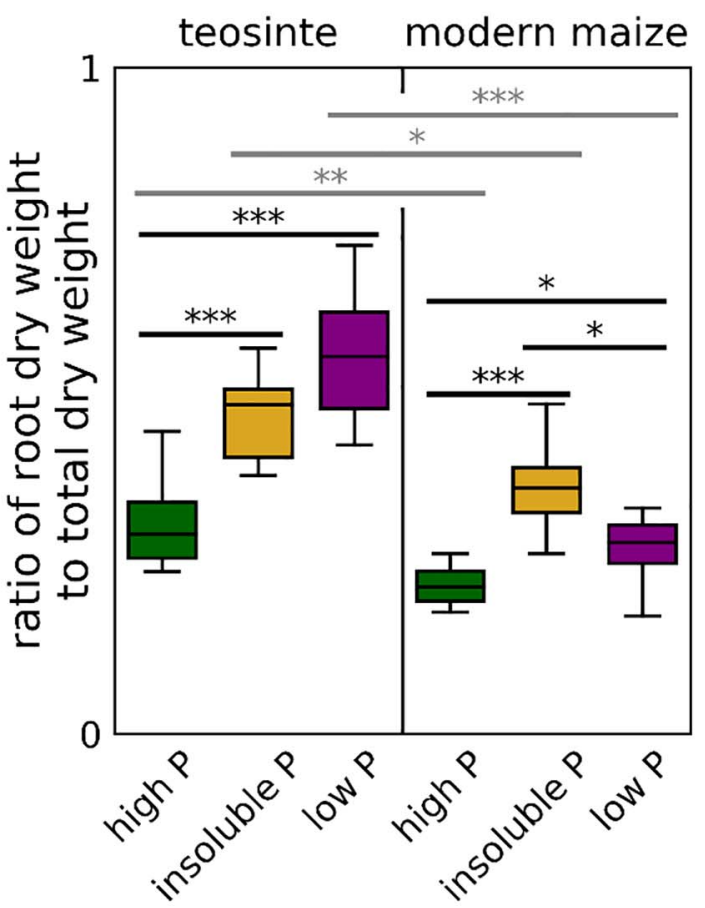

C Shoot P Content

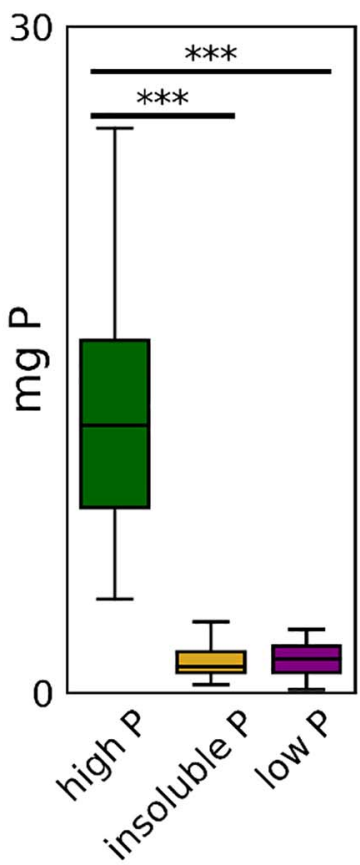

Fig. 1. Plant growth and phosphorus content. A, Total plant biomass. Boxplot showing the total biomass (the combined dry weight of the washed roots and shoots) for each combination of plant accession and phosphate treatment. B, Root biomass proportion. Boxplot showing the ration of root dry weight to total dry weight for each combination of plant accession and phosphate treatment. C, Shoot phosphorus content. Total amount of phosphorus present in the shoots at the end of the experiment (21 days). Statistically significant differences based on Bonferroni corrected $P$ values for pairwise $t$ tests are marked by asterisks: ***, **, and * indicate $P_{\text {adjusted }}<0.001,0.01$, and 0.05 , respectively. 
the teosinte under the low phosphate condition (Fig. 1A). Plant biomass was significantly affected by latitudinal position of the plant in the greenhouse, with plant biomass increasing from north (lower light) to south (higher light) across the greenhouse (Supplementary Fig. S1). We included latitudinal position (which correlated with light exposure) as a variable in our statistical models for this and subsequent analyses.

The proportion of biomass in the roots (root to total biomass ratio) was significantly affected by the phosphate conditions $(P<$ $0.001)$, plant accession $(P=0.0041)$, and the interaction between them $(P=0.0018)$ but not by latitudinal position $(P=0.12)$ (Supplementary Table S3). Teosinte had a greater proportion of biomass in the roots than the modern maize under all conditions $(P=0.003$, $P=0.026$, and $P<0.001$ for high, insoluble, and low phosphate, respectively) (Fig. 1B). The phosphate stressed conditions (low and insoluble phosphate) resulted in a higher proportion of total biomass allocated to roots than under high phosphate conditions for both plant accessions (Fig. 1B). Although no differences between the insoluble and low phosphate conditions were observed for teosinte, insoluble phosphate resulted in a higher allocation of biomass in modern maize.

$\mathrm{P}$ content in the shoots was significantly affected by phosphate availability but did not differ between plant accessions and was not influenced by latitudinal position (Supplementary Table S4). Shoot $\mathrm{P}$ content was the highest $(12.5 \pm 5.6 \mathrm{mg}$ of $\mathrm{P})$ with high phosphate inputs (Fig. 1C). Insoluble and low phosphate conditions resulted in similarly lower shoot $\mathrm{P}$ uptake $(1.4 \pm 0.7$ and $1.5 \pm 0.8 \mathrm{mg}$ of $\mathrm{P}$ for insoluble and low phosphate, respectively).

Phosphate stress increases root exudation and induces changes in exudate composition. Root exudation levels (total organic carbon exuded per gram of root dry weight) significantly increased under phosphate stress, with the insoluble phosphate condition resulting in intermediate exudation between low and high phosphate (Fig. 2A; Supplementary Table S5). This trend was consistent for both plant accessions. Exudation was also affected by plant latitudinal position, with plants toward the south side of the greenhouse (higher light) having lower exudation levels (Supplementary Fig. S2).

Targeted analysis of liquid chromatography-tandem mass spectrometry data identified 86 unique compounds in root exudates across experimental conditions (Supplementary Tables S6 and S7; Fig. 3). Principle component analysis of metabolite profiles showed clear clustering of root exudate compositions of plants grown with high phosphate input and those experiencing phosphate stress (low or insoluble phosphate) (Fig. 2B). Distinctions in metabolite profiles between the two plant accessions were only evident under high phosphate and were driven by the higher exudation of several P-containing compounds by the teosinte (Fig. 3).

The exudation levels of 42 metabolites were significantly affected by the plant accession, phosphate stress, or both $\left(P_{\text {adjusted }}<0.05\right)$ (Supplementary Figs. S3, S4, and S5). Phosphate stress (low or insoluble phosphate) increased exudation of several amino acids and some aromatic compounds (p-coumaric acid, ferulic acid, and nicotinamide) and reduced exudation of several P-containing metabolites by one or both plant accessions (cytidine $5^{\prime}$-monophosphate, glycerophosphocholine, phsophocoline, hexose-phosphates, adenosine-2,3-cyclic-monophosphate, SN-glycero-3-phosphocholine, cytidine $2^{\prime}, 3^{\prime}$-cyclic monophosphate, glycerol 2-phosphate, and $2^{\prime}$ deoxycytidine $5^{\prime}$-monophosphate).

Host selection and phosphate availability drive microbial community composition. Analysis of the prokaryotic 16S and fungal ITS sequences identified 599 prokaryotic and 143 fungal ASVs detected across all samples. Prokaryotic $\alpha$ diversity, as measured by the Shannon diversity index, was significantly influenced
A Organic Carbon Exudation

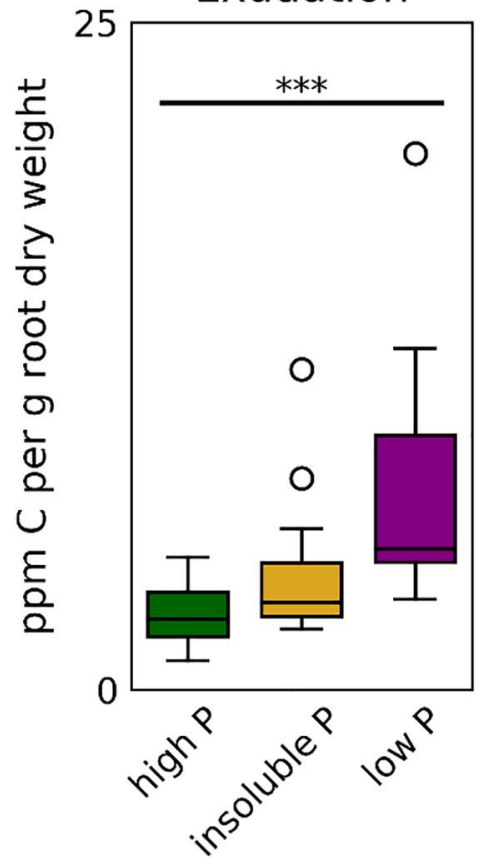

\section{B PCA of Root Exudate Compositions}

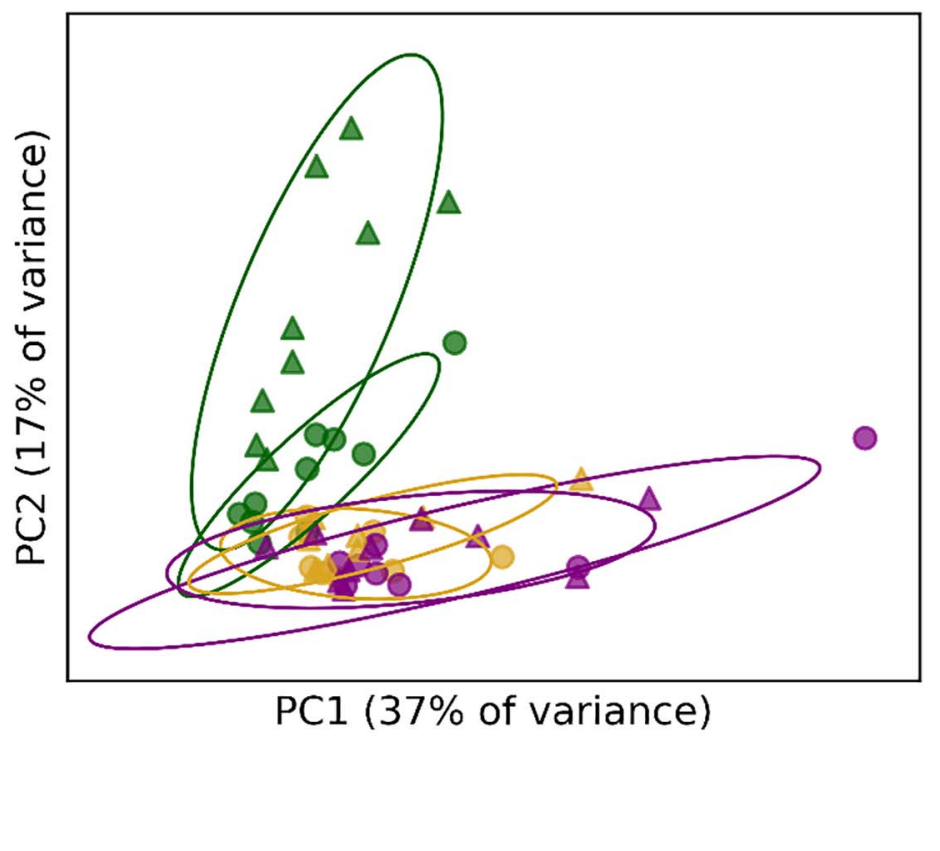

Sample Type modern high P modern insoluble $\mathrm{P}$ modern low $\mathrm{P}$ teosinte $\Delta \quad$ high $\mathrm{P}$ teosinte insoluble $\mathrm{P}$ teosinte $\Delta$ low $\mathrm{P}$

Fig. 2. Root exudate composition. A, Organic carbon exudation. Total organic carbon exuded by the roots during exudate collection (1.5 h), normalized to root dry weight. Statistically significant differences based on Bonferroni corrected $P$ values for pairwise $t$ tests are marked by asterisks: *** indicates $P_{\text {adjusted }}<0.001$. B, Principal component analysis (PCA) of root exudate composition. Each point represents the metabolite profile for exudates from an individual plant replicate. Ellipses represent $95 \%$ confidence intervals. 


\section{Exuded Metabolites}

$\mathrm{N}$-acetyl-glutamic acịd dethiobiotin N-acetyl-serine o-acetyl-serine glucuronic acid

riboflavin glutamine glycine tryptophan

norvaline isoleucine inorleucine allantoin quinic acid choline

p-coumaric acid shikimic acid or isomer glucosaminic acid homoserine threonine/allothreonine glutamic acid 4-hyaroxy-proline trimethyl glycine aspartate 3-sulfino-alanine diethanolamine $\mathrm{N}$-acetyl-5-hydroxytryptamine histidine nicotinamide glucosamain

4-guanidinobutyric acid
2-aminoadipic acid arginine tyrosine lysine guanine adenosine 2'-deoxyadenosinne adenosylmethionine dipalmitoyl carnosine $\mathrm{N}$-alpha-acetyl-asparagine phenylalanine 1-methylnicotinic acid $\mathrm{N}$-alpha-acetyl-lysine raffinose hypoxanthịne alanine adipic acid xanthinne ornithine pipecolic acid
4-Hydroxyquinoline-2-carboxylic acid
thymine thymine

2 '-deoxyguanosine cytidine thymidine inosine

1-methyladenosine methylthioadenosine carboxymethylcysteine $\mathrm{N}$-acetyl-D-galactosamine ferulic acid deoxycarnitine xanthurenic acid 4-aminobutyric acid adenine

galactaric acid

uridine-5-diphospho-N-acetylhexosamines uridine-5-diphosphoglucose cytidine 2',3'-cyclic monophosphate adenosine 2,3 -cyclic-monophosphate guanosine 3 ', 5 '-cyclic monophosphate hexose phosphates cytidine 5'-monophosphate 2'-deoxycytidine 5'-monophosphate phosphocholine glycerophosphocholine

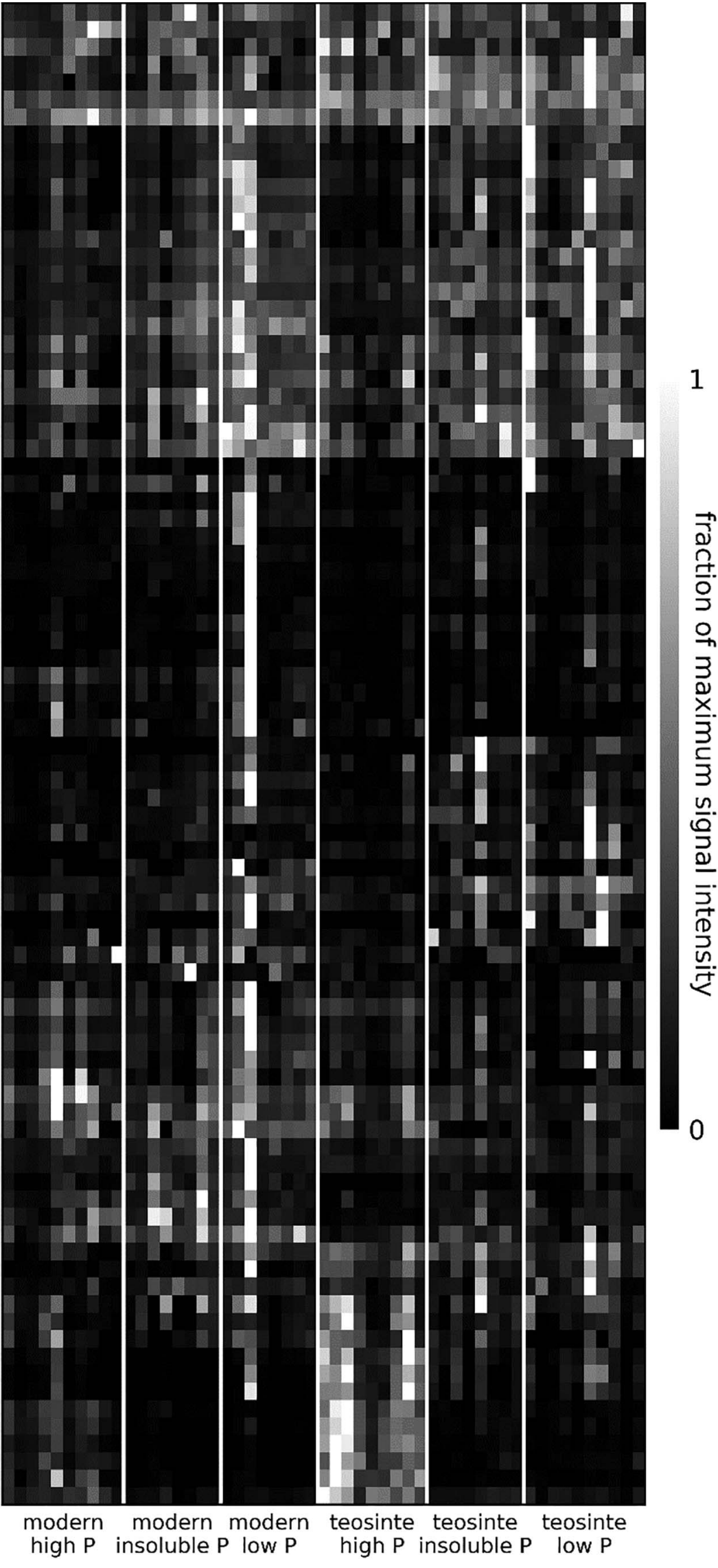


by host plant accession and compartment (root or rhizosphere soil) but was not significantly driven by phosphate availability (Supplementary Table S8). Prokaryotic $\alpha$ diversity was higher in the modern maize accession than in teosinte, and in the rhizosphere compared with the root (endosphere and rhizoplane combined). In contrast, of all the factors tested, only compartment had a significant impact on fungal $\alpha$ diversity (Supplementary Table S9), with the endosphere and rhizoplane having significantly higher diversity than rhizosphere samples.

Both the prokaryotic and fungal microbial community compositions were significantly influenced by the compartment, plant accession, phosphate availability, and latitudinal position (Supplementary Tables S10 and S11). Compartment, plant accession, phosphate, and latitudinal position, accounted for $6.7,4.1,3.8$, and $3.4 \%$ of variance, respectively, in the prokaryotic community composition. Interaction effects were also detected between plant accession and phosphate availability, and between plant accession and compartment, accounting for 3.0 and $1.9 \%$ of variance, respectively. PCoA of CLR-transformed microbial community composition data showed clustering of the prokaryotic microbial communities by compartment and plant accession (Fig. 4A). For the fungal community, compartment, plant accession, phosphate, and latitudinal position in the greenhouse accounted for $4.3,9.3,3.4$, and $2.9 \%$ of variance, respectively (Fig. 4B). Interaction effects between plant accession and phosphate and between plant accession and compartment were also significant, accounting for 2.8 and $1.6 \%$ of variance, respectively.

Indicator species analysis identified individual ASVs associated with different sample groups based on ASV presence or absence (Supplementary Tables S12 and S13). For plant accessions, 48 prokaryotic ASVs $(8.1 \%)$ were indicators for modern maize samples, and 8 prokaryotic ASVs (1.8\%) were indicators for teosinte. The most abundant of these were 16S_ASV_5 (4.7\% overall relative abundance), an indicator for the teosinte, and 16S_ASV_9 $(2.7 \%$ overall relative abundance), an indicator for the modern maize. Both of these ASVs were identified as Pseudomonas. In all, 73 prokaryotic prokaryotic ASVs (12.5\%) were indicators for rhizosphere soil samples and $17(3.3 \%)$ were indicators for root samples. Indicator species analysis did not identify any individual prokaryotic ASVs associated with phosphate condition or latitudinal position in the greenhouse. Similarly, for the fungal community, eight fungal ASVs (5.6\%) were indicators for modern maize samples and nine fungal ASVs $(6.3 \%)$ were indicators for teosinte. These included several ASVs identified as Sarocladium zeae (ITS_ASV_1, an indicator for the modern maize, and ITS_ASV_6, ITS_ASV_11, ITS_ASV_17, and ITS_ASV_22, indicators for the teosinte). Twenty fungal ASVs (14\%) were indicators for root samples but no fungal ASVs were identified as indicators for rhizosphere samples, or for phosphate condition or latitudinal position.

In contrast the indicator species analysis, which did not identify ASVs associated with phosphate conditions based on presence or absence, differential abundance analyses identified several ASVs enriched under some phosphate conditions compared with others (Fig. 5; Supplementary Table S14). Across the sample set, five bacterial ASVs were more abundant under low phosphate conditions and two were more abundant under high phosphate (Fig. 5A). These included one, 16S_ASV_3, a member of the Enterobacteriaceae family, that represented an average relative abundance of $5.2 \%$ across samples and was highly enriched in the low phosphate condition compared with high. Two others, 16S_ASV_28 and 16S_ASV_38, had mean relative abundances between 0.1 and $1 \%$. 16S_ASV_28, identified as a Rhizobium sp., was more abundant under low phosphate compared with high, and 16S_ASV_38, identified as a Massilia sp., was more abundant under high phosphate compared with low (Supplementary Table S14). Six fungal ASVs were differentially abundant between phosphate conditions, all having average relative abundances between 0.1 and $1 \%$ (Fig. 5B.)

In order to identify differential microbial responses to phosphate between the two plant accessions, we also performed differential abundance analyses on the teosinte samples and modern maize samples separately (Fig. 5C to F; Supplementary Table S14). Within the teosinte samples, six bacterial ASVs were differentially

\section{A Prokaryotic Community Composition}

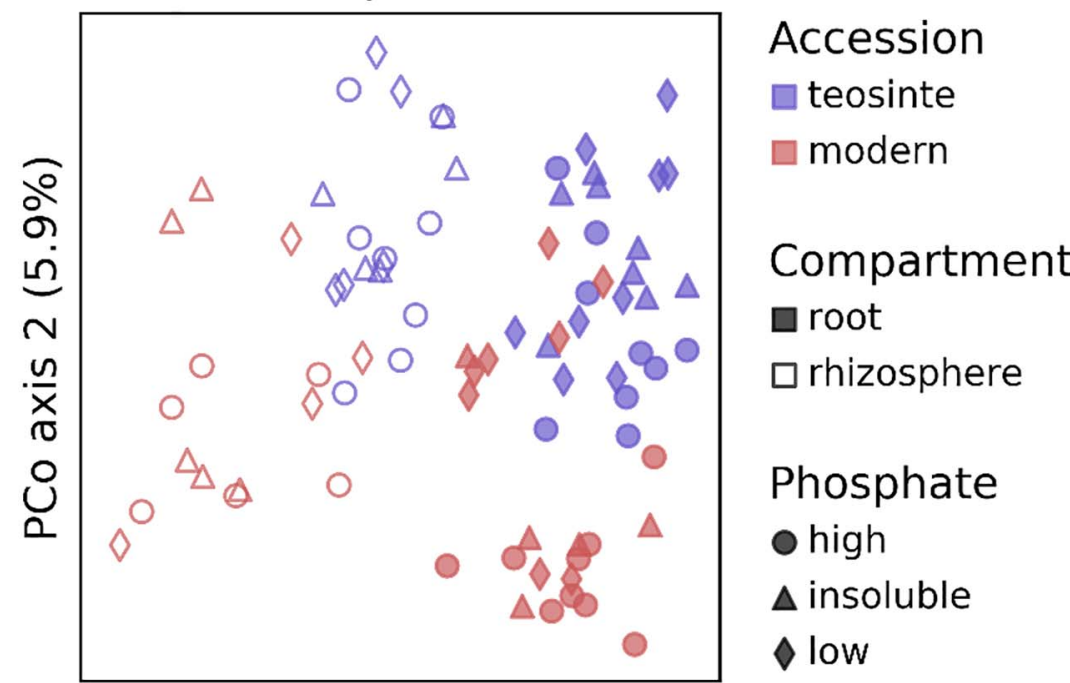

PCo axis $1(8.0 \%)$

\section{B Fungal Community Composition}

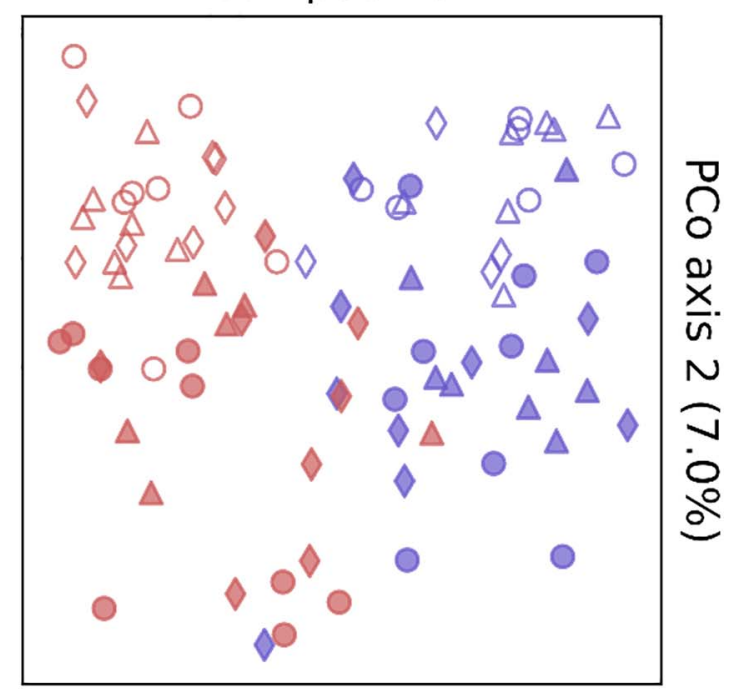

PCo axis 1 (13.5\%)

Fig. 4. Microbial community compositions. A, Principal coordinate (PCo) analysis of the prokaryotic community composition using Bray-Curtis dissimilarity. B, PCo analysis of the fungal community composition using Bray-Curtis dissimilarity. Each point represents the microbial community composition for an individual soil or root sample. 


\section{Differential Abundance Ternary Plots}

Prokaryotic ASVs

Fungal ASVs

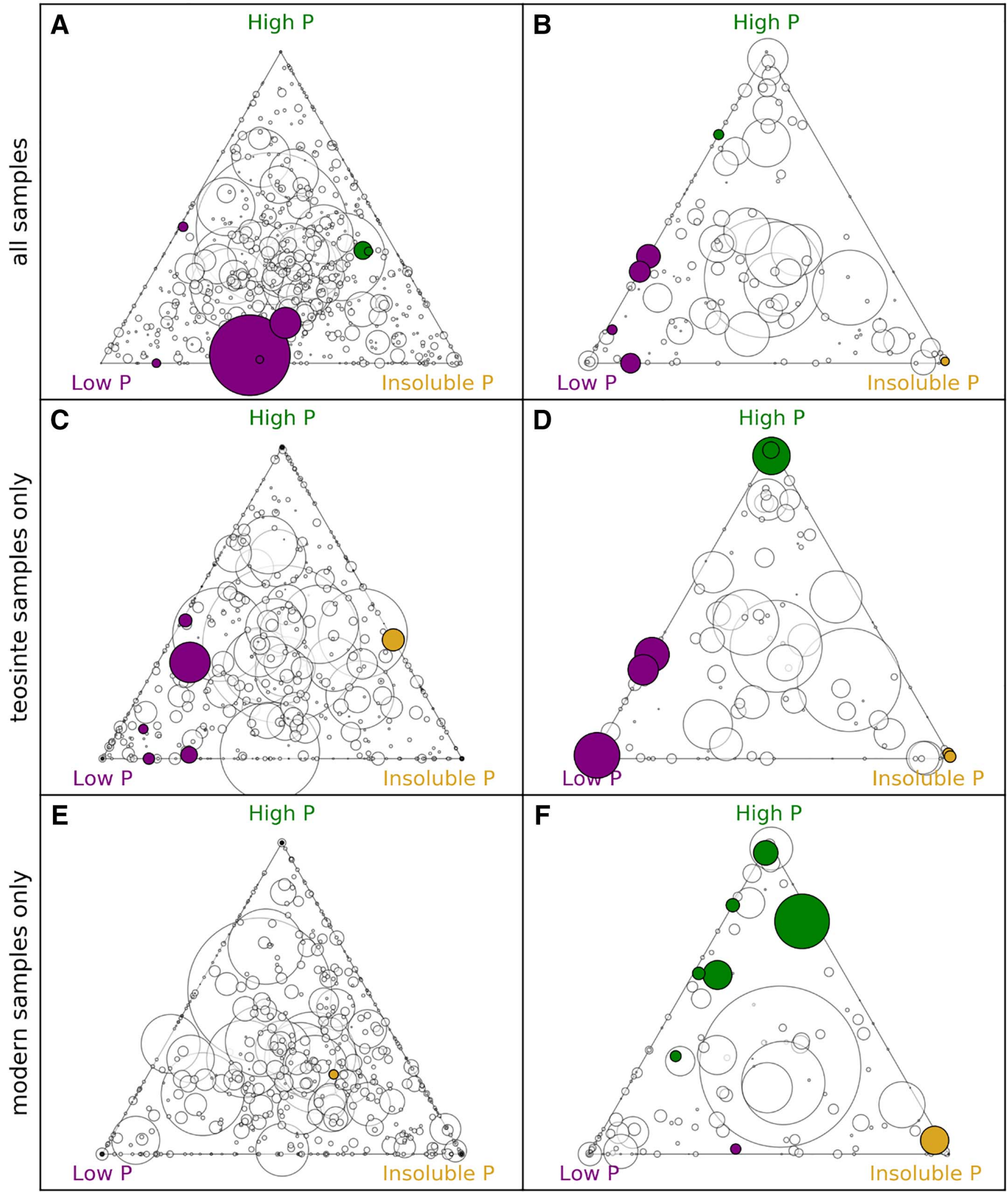

Fig. 5. Amplicon sequence variant (ASV) differential abundance. Ternary plots showing individual ASVs as circles. Circle size is proportional to average relative abundance. Position indicates relative abundance across the three phosphate conditions. Filled circles indicate ASVs that were significantly differentially abundant. Fill color indicates which condition under which the ASV was more abundant: green $=$ high phosphorus $(P)$, purple $=$ low $P$, and yellow = insoluble P. A and B, Analysis of all rhizosphere and root samples from both plant accessions. C and D, Analysis of teosinte samples only. E and F, Analysis of modern maize samples only. See Supplementary Table S14 for information on individual differentially abundant ASVs. 
abundant between the phosphate conditions (Fig. 5C). Three of these were the same ASVs identified in the analysis of all samples above, while three were specifically enriched under either low or insoluble phosphate conditions for teosinte only (Supplementary Table S14). The most abundant of these $(0.7 \%$ relative abundance across all samples, $1.3 \%$ across teosinte samples) was 16S_ASV_22, identified as a Paenibacillus sp., which was most abundant under low phosphate condition. Notably, this was also an indicator ASV for teosinte. In contrast, within the modern maize samples, only one ASV (ASV_159, identified as a Pedobacter sp.) was differentially abundant between phosphate conditions, with the highest abundance with insoluble phosphate (Fig. 5E). Within the fungal ASVs, seven were differentially abundant among the phosphate conditions for the teosinte samples (Fig. 5D), and eight were differentially abundant for the modern maize samples (Fig. 5F). There was no overlap of the teosinte differentially abundant ASVs and the modern maize differentially abundant ASV, highlighting the differences between the fungal communities associated with these two plant accessions.

Overall microbial community composition and root exudate metabolite profiles were not significantly correlated for bacterial or fungal communities in either the root or rhizosphere samples. Biomass properties were significantly correlated with overall microbial community composition for the teosinte root microbiomes but not for the modern maize or rhizosphere microbiomes. Total teosinte biomass correlated with the root fungal community $(r=24, P=$ $0.035)$, and teosinte shoot $\mathrm{P}$ content correlated with the rood bacterial community $(r=0.20, P=0.034)$.

Some individual ASVs were significantly correlated with individual root exudate metabolites, total biomass, or biomass $\mathrm{P}$ content.

\section{Phosphate Solubiliers}

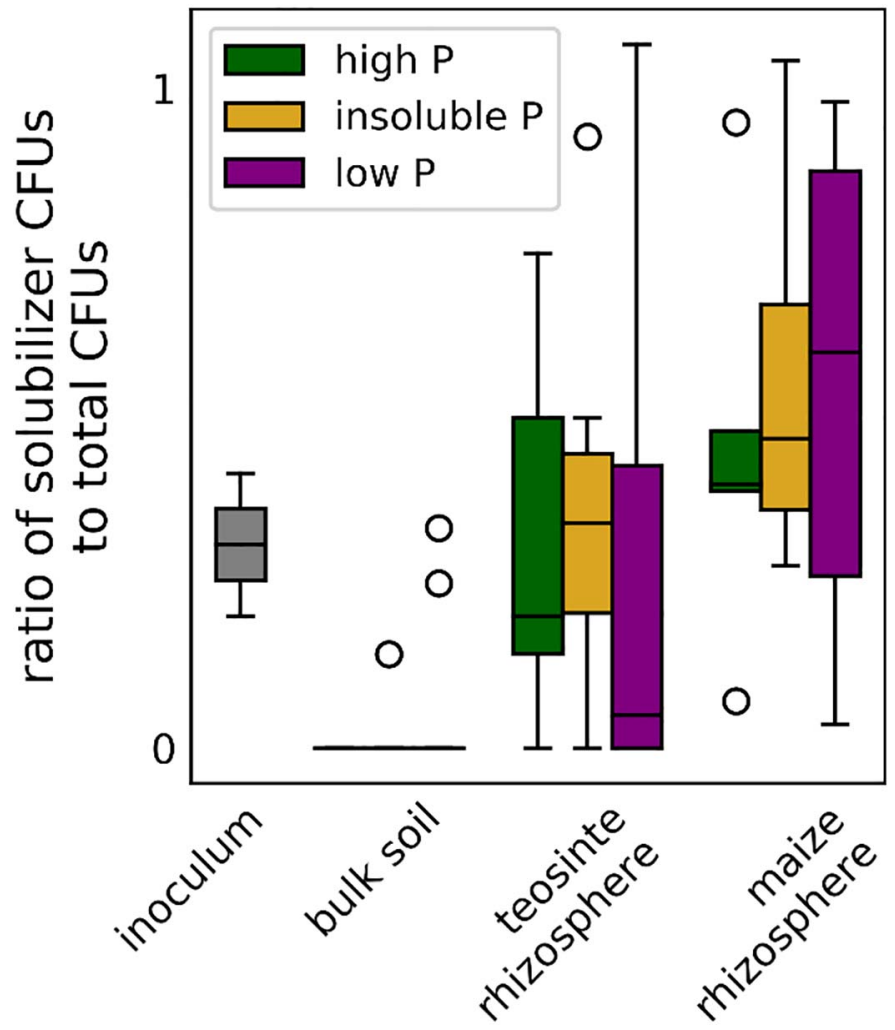

Fig. 6. Phosphate solubilizers in the rhizosphere. Boxplot showing the proportion of phosphate solubilizers recovered in plate assays from rhizosphere soil samples. $\mathrm{P}=$ phosphorus.
Within plant root microbial communities, one prokaryote $\left(16 \mathrm{~S}_{-}\right.$ ASV_31, identified as a Massilia sp.) was positively correlated with hexose phosphates, while another (16S_ASV_75, identified as a Sulfuricella sp.) was correlated with 14 metabolites, including several nucelosides (adenosine, 2'-deoxyadenosine, 1-methyladenosine, methylthioadenosine, cytidine, uridine, and inosine). In the rhizosphere, one fungal ASVs (ITS_ASV_1, identified as S. zeae) had significant positive correlations with four metabolites (adenosine, carnitine, deoxycarnitine, and 4-hydroxy-proline). Two prokaryotic ASVs were correlated between the teosinte root microbial community and total biomass. 16S_ASV_153 (identified as a Aciditerrimonas sp.) was positively correlated with biomass, while 16S_ASV_269 (identified as a Staphylococcus sp.) was negatively correlated with biomass. One ASV (16S_ASV_129, identified as a Sphingomonas $\mathrm{sp}$.) was significantly negatively correlated with shoot $\mathrm{P}$ content in the modern maize root microbiome. Interestingly, this ASV was an indicator species for the root compartment (Supplementary Table S13) and was also differentially abundant, with greater abundance under low phosphate conditions (Supplementary Table S14). Thus, this correlation may represent the variation of both variables with phosphate availability because biomass decreased under low phosphate when this ASV increased.

Phosphate-solubilizing microorganisms are present in the rhizosphere. Phosphate solubilizers were detected in the initial inoculum but not in most bulk soil samples at the end of the experiment, which was dominated by nonsolubilizers capable of growing with $\mathrm{KH}_{2} \mathrm{PO}_{4}$ but not $\mathrm{FePO}_{4} \cdot 2 \mathrm{H}_{2} \mathrm{O}$ as the $\mathrm{P}$ source (Fig. 6). Phosphate solubilizers were found to be a high proportion of culturable rhizosphere microorganisms for both plant accessions under all growth conditions. Levels of phosphate solubilizers in the plant rhizosphere detected with our method were highly variable within sample groups, and no significant differences were detected between phosphate conditions or plant accession.

\section{DISCUSSION}

Our goal was to better understand how phosphate availability regulates root exudation and rhizosphere and root microbial community assembly in two maize accessions separated by 10,000 years of crop improvements. We found that phosphate availability partly regulates root exudation and microbial community composition in both a teosinte and modern maize. Our result showing shifts in root exudate composition in response to phosphate stress are consistent with previous studies, which have found changes in the root exudate composition of maize and other plants in response to nutrient limitations, including phosphate limitation (Carvalhais et al. 2011; Edayilam et al. 2018). However, to our knowledge, previous studies have not investigated parallel responses in root exudation from a domesticated plant and its wild relative. Under phosphate replete conditions, exudate compositions of the two plant accessions differed, driven largely by high exudation of P-containing metabolites by the teosinte. Despite these differences under phosphate replete conditions, shifts in both the quantity and composition of exudates under phosphate stress revealed a similar response by both the teosinte and the modern maize cultivar. This suggests that, contrary to our original hypothesis, the mechanisms of response to phosphate stress may have been conserved in the transition from teosinte to modern maize, at least in terms of root exudation. However, because we only examined a single accession of each type, we cannot say how broadly phosphate stress responses have been conserved after breeding.

Plants that experienced phosphate stress increased exudation of several metabolites, including several amino acids and aromatic compounds. Some of these compounds may be involved in direct 
solubilization of insoluble phosphate. For instance, aspartate, which was exuded at higher levels by both teosinte and maize under phosphate stress, has been shown to form complexes with metal ions, and could be involved in direct solubilization via this complex formation mechanism (Edayilam et al. 2018).

Both amino acids and aromatic compounds could also be involved in recruitment of specific microorganisms, including phosphate solubilizers, to the rhizosphere. Rhizosphere-associated microorganisms have different abilities to uptake and use different root exudate metabolites. Shikimic acid and quinic acid, two compounds that increased under phosphate stress in our study, have been shown to be preferentially taken up by microorganisms that were positively correlated with root growth (Zhalnina et al. 2018). This suggest that increased exudation of these and other root exudate metabolites in response to stress may be shaping the microbial community by preferentially stimulating the growth of some groups of organisms. We did detect correlations between individual ASVs and individual metabolites. However, because this was a correlation and not a mechanistic analysis, we are not able to conclude whether specific metabolites caused observed changes in microbial abundance.

Both prokaryotic and fungal community compositions were driven by compartment and plant type and, to a lesser extent, by phosphate availability. We also found a significant interaction effect between plant type and phosphate availability, suggesting differences in how the teosinte and maize actively recruit and assemble their rhizosphere and root microbial communities. Two recent field studies investigating the effects of phosphate fertilization and availability on maize rhizosphere microbiomes found phosphate conditions to be primary drivers of microbial community composition (Gomes et al. 2018; Silva et al. 2017). Our result in a controlled environment with more acute changes in phosphate levels showed significant but relatively small effects of phosphate availability on rhizosphere microbiomes. These studies (Gomes et al. 2018; Silva et al. 2017) addressed long-term effects of phosphate availability on soil microbial communities, whereas our approach focused on the selective effects of the plant in the early stages of growth response to different phosphate conditions, with all cultivars recruiting from the same initial microbial community inoculum. Gomes et al. (2018) also found that compartment (root versus rhizosphere) had a greater effect than maize genotype on both prokaryotic and fungal microbial communities. Although our study showed a similar pattern for prokaryotes associated with maize and teosinte, we found that, for fungi, the plant type had a larger impact than compartment, highlighting the potential importance of differences between maize and teosinte in recruiting fungal partners. Differences in the $\alpha$ diversity of both prokaryotic and fungal communities were driven largely by compartment (root versus rhizosphere). For the prokaryotic community, diversity was greater in the rhizosphere than the root, which is consistent with previous findings showing higher prokaryotic diversity farther from the selective pressure of the plant (Brisson et al. 2019; Coleman-Derr et al. 2016; Peiffer et al. 2013). The reverse pattern for fungi (higher diversity in the roots than in the rhizosphere) is also consistent with previously reported gradients of fungal diversity based on proximity to the roots of maize and agave (Brisson et al. 2019; Coleman-Derr et al. 2016).

Differential abundance analysis revealed individual ASVs enriched under different phosphate conditions. Overall, the teosinte system had more differentially abundant prokaryotic ASVs than found in the modern maize, suggesting more responsiveness to phosphate conditions in this system. Castrillo et al. (2017) found that $A$. thaliana differentially recruited specific bacteria from a synthetic community in response to phosphate limitation but that mutations in A. thaliana phosphate stress-response genes affected the enrichment of these bacteria. Several of the prokaryotic ASVs in our study that were enriched under phosphate stress were identified as belonging to genera that have been associated with phosphate solubilization and plant growth promotion, including Rhizobium, Massilia, Pseudomonas, and Paenibacillus (Grady et al. 2016; Oteino et al. 2015; Sridevi and Mallaiah 2009; Zheng et al. 2017). Another ASV enriched under low phosphate conditions, particularly in teosinte, was identified as Xanthomonas, a genus containing many plant pathogens (Niño-Liu et al. 2006). This could represent opportunistic infection of plants under stress conditions. In both the teosinte and maize systems, similar numbers of fungal ASVs were differentially abundant but there was no overlap between the groups of ASVs that were differentially abundant in these two systems, highlighting the influence of plant accession on fungal communities, as was seen in the PCoA analysis (Figs. 4 and 5). Similar to prokaryotic ASVs, differentially abundant fungal ASVs included some genera known to include phosphate solubilizers and plant growth promoters (Rhodotorula and Mortierella) (Botha 2011; Ozimek and Hanaka 2021) and others containing known pathogens (Alternaria, Fusarium, and Gibberella) (Desjardins and Plattner 2003; Ma et al. 2013; Thomma 2003).

Fungi are known to be critically important for plant phosphate acquisition, particularly under phosphate-limited conditions. In our study, we observed a strong effect of plant accession on fungal microbial community in both the PCoA analysis (Fig. 4B) and the differential abundance analysis (Fig. 5; Supplementary Table S14). Mycorrhizal fungi have long been studied for their ability to access limited phosphate resources and make phosphate available to their symbiotic plant partners (Bucher 2007; Karandashov and Bucher 2005; Sanders and Tinker 1971), and phosphate fertilization has been shown to inhibit the formation of arbuscular mycorrhizal associations (Breuillin et al. 2010). Nonmycorrhizal fungi have also been shown to have important phosphate-solubilizing and plantgrowth-promoting activity (Agnihotri 1970; Altomare et al. 1999; Pradhan and Sukla 2006).

Rhizosphere samples from all growth conditions in our study harbored phosphate-solubilizing microorganisms, while samples from unplanted control pots did not. The proportion of solubilizers in rhizosphere soils were similar across both plant accessions and all growth conditions, suggesting that plants were not selectively recruiting solubilizers to the rhizosphere in response to phosphate stress. Gomes et al. (2018) speculated that phosphate solubilizers might not be selectively recruited by maize based on the genera identified in their microbial community analysis of maize rhizospheres. The phosphate solubilization assay in our study provides evidence that this might be the case. Our results also contradict our initial hypothesis that teosinte would recruit phosphate solubilizers more effectively than maize under phosphate stress. Instead, maize and teosinte appear to recruit phosphate solubilizers constitutively and at similar levels to each other. However, the high variability within sample groups may mask underlying differences, and other methods such as quantitative PCR (qPCR), targeting phosphate-solubilization-related genes, could shed more light on this question in future studies. The method used here also only detects microorganisms that can be cultivated on plates, excluding other potentially important community members. Further study is also necessary to determine whether phosphate solubilizers from different plant accessions or growth conditions differ in plant growth promotion.

Along with plant accession and phosphate treatment, the latitudinal position of the plants in the greenhouse also had significant unexpected impacts in our study. Our study utilized a fully randomized design, a common experimental design for controlled greenhouse studies. This type of design has been used to study the effects on maize of a variety of treatments such as inoculation with diazotrophic bacteria (Zeffa et al. 2019), application of green 
manure and natural phosphate source (de Medeiros et al. 2019), and amendment with biochar (Rajkovich et al. 2012). Although the latitudinal light gradient observed in our experiment was not anticipated, the high replication in our experiment (10 replicates per each combination of plant accession and treatment) allowed us to incorporate its effects into our statistical analyses. By including latitudinal position as a variable in our models, we were able to not only separate its effects from those of plant accession and phosphate treatment but also consider the robustness of those effects across the light gradient. We found that dependent variables such as plant biomass and organic carbon exudation were significantly affected by latitudinal position but the impacts of phosphate treatment were consistent along the latitudinal gradient (Supplementary Figs. S1 and S2).

The results of this study expand our understanding of the response of both wild and domesticated Z. mays to phosphate stress. The shifts in both root exudate and microbiome compositions demonstrated a consistent response to phosphate stress. However, we did not detect increased recruitment of phosphate solubilizers. Further studies could investigate microbial community changes through other functional assays as well as shifts in functional potential through qPCR targeting phosphate-solubilization-related genes or metagenomics. Understanding these responses is a necessary step toward enabling more sustainable agricultural practices with regard to phosphate availability and fertilization.

\section{ACKNOWLEDGMENTS}

We thank Dupont Pioneer for sharing seed from the ERA panel.

\section{LITERATURE CITED}

Agnihotri, V. P. 1970. Solubilization of insoluble phosphates by some soil fungi isolated from nursery seedbeds. Can. J. Microbiol. 16:877-880.

Aitchison, J. 1982. The statistical-analysis of compositional data. J. R. Stat. Soc. B 44:139-177.

Alori, E. T., Glick, B. R., and Babalola, O. O. 2017. Microbial phosphorus solubilization and its potential for use in sustainable agriculture. Front. Microbiol. 8:971.

Altomare, C., Norvell, W. A., Bjorkman, T., and Harman, G. E. 1999. Solubilization of phosphates and micronutrients by the plant-growthpromoting and biocontrol fungus Trichoderma harzianum Rifai 1295-22. Appl. Environ. Microbiol. 65:2926-2933.

Apprill, A., McNally, S., Parsons, R., and Weber, L. 2015. Minor revision to V4 region SSU rRNA 806R gene primer greatly increases detection of SAR11 bacterioplankton. Aquat. Microb. Ecol. 75:129-137.

Arcand, M. M., and Schneider, K. D. 2006. Plant- and microbial-based mechanisms to improve the agronomic effectiveness of phosphate rock: A review. An. Acad. Bras. Cienc. 78:791-807.

Barillot, C. D. C., Sarde, C. O., Bert, V., Tarnaud, E., and Cochet, N. 2013. A standardized method for the sampling of rhizosphere and rhizoplan soil bacteria associated to a herbaceous root system. Ann. Microbiol. 63: 471-476.

Botha, A. 2011. The importance and ecology of yeasts in soil. Soil Biol. Biochem. 43:1-8.

Bowen, B. P., and Northen, T. R. 2010. Dealing with the unknown: Metabolomics and metabolite atlases. J. Am. Soc. Mass Spectrom. 21: 1471-1476.

Breuillin, F., Schramm, J., Hajirezaei, M., Ahkami, A., Favre, P., Druege, U., Hause, B., Bucher, M., Kretzschmar, T., Bossolini, E., Kuhlemeier, C., Martinoia, E., Franken, P., Scholz, U., and Reinhardt, D. 2010. Phosphate systemically inhibits development of arbuscular mycorrhiza in Petunia hybrida and represses genes involved in mycorrhizal functioning. Plant J. 64:1002-1017.

Brisson, V. L., Schmidt, J. E., Northen, T. R., Vogel, J. P., and Gaudin, A. C. M. 2019. Impacts of maize domestication and breeding on rhizosphere microbial community recruitment from a nutrient depleted agricultural soil. Sci. Rep. 9:15611.
Brisson, V. L., Zhuang, W. Q., and Alvarez-Cohen, L. 2016. Bioleaching of rare earth elements from monazite sand. Biotechnol. Bioeng. 113:339-348.

Bucher, M. 2007. Functional biology of plant phosphate uptake at root and mycorrhiza interfaces. New Phytol. 173:11-26.

Callahan, B. J., McMurdie, P. J., Rosen, M. J., Han, A. W., Johnson, A. J. A., and Holmes, S. P. 2016. DADA2: High-resolution sample inference from Illumina amplicon data. Nat. Methods 13:581-583.

Carvalhais, L. C., Dennis, P. G., Fedoseyenko, D., Hajirezaei, M. R., Borriss, R., and von Wiren, N. 2011. Root exudation of sugars, amino acids, and organic acids by maize as affected by nitrogen, phosphorus, potassium, and iron deficiency. J. Plant Nutr. Soil Sci. 174:3-11.

Castrillo, G., Teixeira, P., Paredes, S. H., Law, T. F., de Lorenzo, L., Feltcher, M. E., Finkel, O. M., Breakfield, N. W., Mieczkowski, P., Jones, C. D., Paz-Ares, J., and Dangl, J. L. 2017. Root microbiota drive direct integration of phosphate stress and immunity. Nature 543:513-518.

Cheng, N., Peng, Y. J., Kong, Y. L., Li, J. J., and Sun, C. X. 2018. Combined effects of biochar addition and nitrogen fertilizer reduction on the rhizosphere metabolomics of maize (Zea mays L.) seedlings. Plant Soil 433:19-35.

Coleman-Derr, D., Desgarennes, D., Fonseca-Garcia, C., Gross, S., Clingenpeel, S., Woyke, T., North, G., Visel, A., Partida-Martinez, L. P., and Tringe, S. G. 2016. Plant compartment and biogeography affect microbiome composition in cultivated and native Agave species. New Phytol. 209:798-811.

De Cáceres, M., and Legendre, P. 2009. Associations between species and groups of sites: Indices and statistical inference. Ecology 90:3566-3574

de Medeiros, E. V., Silva, A. O., Duda, G. P., dos Santos, U. J., and de Souza, A. J. 2019. The combination of Arachis pintoi green manure and natural phosphate improves maize growth, soil microbial community structure and enzymatic activities. Plant Soil 435:175-185.

Desjardins, A. E., and Plattner, R. D. 2003. Diverse traits for pathogen fitness in Gibberella zeae. Can. J. Plant Pathol. 25:21-27.

Doebley, J., Stec, A., and Hubbard, L. 1997. The evolution of apical dominance in maize. Nature 386:485-488.

Edayilam, N., Montgomery, D., Ferguson, B., Maroli, A. S., Martinez, N., Powell, B. A., and Tharayil, N. 2018. Phosphorus stress-induced changes in plant root exudation could potentially facilitate uranium mobilization from stable mineral forms. Environ. Sci. Technol. 52:7652-7662.

Emmett, B. D., Buckley, D. H., Smith, M. E., and Drinkwater, L. E. 2018. Eighty years of maize breeding alters plant nitrogen acquisition but not rhizosphere bacterial community composition. Plant Soil 431:53-69.

Gardes, M., and Bruns, T. D. 1993. Its primers with enhanced specificity for basidiomycetes-Application to the identification of mycorrhizae and rusts. Mol. Ecol. 2:113-118.

Gloor, G. B., Macklaim, J. M., Pawlowsky-Glahn, V., and Egozcue, J. J. 2017. Microbiome datasets are compositional: And this is not optional. Front. Microbiol. 8:2224.

Gomes, E. A., Lana, U. G. P., Quensen, J. F., de Sousa, S. M., Oliveira, C. A., Guo, J. R., Guimaraes, L. J. M., and Tiedje, J. M. 2018. Rootassociated microbiome of maize genotypes with contrasting phosphorus use efficiency. Phytobiomes J. 2:129-137.

Grady, E. N., MacDonald, J., Liu, L., Richman, A., and Yuan, Z. C. 2016. Current knowledge and perspectives of Paenibacillus: A review. Microb. Cell Fact. 15:203.

Hinsinger, P. 2001. Bioavailability of soil inorganic P in the rhizosphere as affected by root-induced chemical changes: A review. Plant Soil 237: 173-195.

Huang, A. C. C., Jiang, T., Liu, Y. X., Bai, Y. C., Reed, J., Qu, B. Y., Goossens, A., Nutzmann, H. W., Bai, Y., and Osbourn, A. 2019. A specialized metabolic network selectively modulates Arabidopsis root microbiota. Science 364:eaau6389.

Huang, C.-Y. L., and Schulte, E. E. 1985. Digestion of plant-tissue for analysis by ICP emission-spectroscopy. Commun. Soil Sci. Plant Anal. 16: 943-958.

Jacoby, R., Peukert, M., Succurro, A., Koprivova, A., and Kopriva, S. 2017. The role of soil microorganisms in plant mineral nutrition-Current knowledge and future directions. Front. Plant Sci. 8:1617.

Karandashov, V., and Bucher, M. 2005. Symbiotic phosphate transport in arbuscular mycorrhizas. Trends Plant Sci. 10:22-29.

Liu, Z. G., Liu, X. S., Craft, E. J., Yuan, L. X., Cheng, L. Y., Mi, G. H., and Chen, F. J. 2018. Physiological and genetic analysis for maize root characters and yield in response to low phosphorus stress. Breed. Sci. 68: 268-277. 
Love, M. I., Huber, W., and Anders, S. 2014. Moderated estimation of fold change and dispersion for RNA-seq data with DESeq2. Genome Biol. 15:550.

Ma, L. J., Geiser, D. M., Proctor, R. H., Rooney, A. P., O’Donnell, K., Trail, F., Gardiner, D. M., Manners, J. M., and Kazan, K. 2013. Fusarium pathogenomics. Annu. Rev. Microbiol. 67:399-416.

McMurdie, P. J., and Holmes, S. 2013. phyloseq: An R package for reproducible interactive analysis and graphics of microbiome census data. PLoS One 8:e61217.

Murashige, T., and Skoog, F. 1962. A revised medium for rapid growth and bio assays with tobacco tissue cultures. Physiol. Plant. 15:473-497.

Nautiyal, C. S. 1999. An efficient microbiological growth medium for screening phosphate solubilizing microorganisms. FEMS Microbiol. Lett. 170:265-270.

Niño-Liu, D. O., Ronald, P. C., and Bogdanove, A. J. 2006. Xanthomonas oryzae pathovars: Model pathogens of a model crop. Mol. Plant Pathol. 7: 303-324.

Oteino, N., Lally, R. D., Kiwanuka, S., Lloyd, A., Ryan, D., Germaine, K. J., and Dowling, D. N. 2015. Plant growth promotion induced by phosphate solubilizing endophytic Pseudomonas isolates. Front. Microbiol. 6:745.

Ozimek, E., and Hanaka, A. 2021. Mortierella species as the plant growthpromoting fungi present in the agricultural soils. Agriculture 11:7.

Parada, A. E., Needham, D. M., and Fuhrman, J. A. 2016. Every base matters: Assessing small subunit rRNA primers for marine microbiomes with mock communities, time series and global field samples. Environ. Microbiol. 18:1403-1414.

Peiffer, J. A., Spor, A., Koren, O., Jin, Z., Tringe, S. G., Dangl, J. L., Buckler, E. S., and Ley, R. E. 2013. Diversity and heritability of the maize rhizosphere microbiome under field conditions. Proc. Natl. Acad. Sci. U.S.A. 110:6548-6553.

Pérez-Jaramillo, J. E., Mendes, R., and Raaijmakers, J. M. 2016. Impact of plant domestication on rhizosphere microbiome assembly and functions. Plant Mol. Biol. 90:635-644.

Pii, Y., Mimmo, T., Tomasi, N., Terzano, R., Cesco, S., and Crecchio, C. 2015. Microbial interactions in the rhizosphere: Beneficial influences of plant growth-promoting rhizobacteria on nutrient acquisition process. A review. Biol. Fertil. Soils 51:403-415.

Pikovskaya, R. I. 1948. Mobilization of phosphorus in soil in connection with the vital activity of some microbial species. Mikrobiologiya 17:362-370.

Porter, S. S., and Sachs, J. L. 2020. Agriculture and the disruption of plantmicrobial symbiosis. Trends Ecol. Evol. 35:426-439.

Pradhan, N., and Sukla, L. B. 2006. Solubilization of inorganic phosphates by fungi isolated from agriculture soil. Afr. J. Biotechnol. 5:850-854.

Rajkovich, S., Enders, A., Hanley, K., Hyland, C., Zimmerman, A. R., and Lehmann, J. 2012. Corn growth and nitrogen nutrition after additions of biochars with varying properties to a temperate soil. Biol. Fertil. Soils 48 : 271-284.

Ranum, P., Peña-Rosas, J. P., and Garcia-Casal, M. N. 2014. Global maize production, utilization, and consumption. Ann. N. Y. Acad. Sci. 1312:105-112.

Rodríguez, H., and Fraga, R. 1999. Phosphate solubilizing bacteria and their role in plant growth promotion. Biotechnol. Adv. 17:319-339.

Sanders, F. E., and Tinker, P. B. 1971. Mechanism of absorption of phosphate from soil by endogone mycorrhizas. Nature 233:278-279.

Schmidt, J. E., Bowles, T. M., and Gaudin, A. C. M. 2016. Using ancient traits to convert soil health into crop yield: Impact of selection on maize root and rhizosphere function. Front. Plant Sci. 7:373.
Schmidt, J. E., Poret-Peterson, A., Lowry, C. J., and Gaudin, A. C. M. 2020a. Has agricultural intensification impacted maize root traits and rhizosphere interactions related to organic $\mathrm{N}$ acquisition? AoB Plants 12: plaa026.

Schmidt, J. E., Rodrigues, J. L. M., Brisson, V. L., Kent, A., and Gaudin, A. C. M. 2020b. Impacts of directed evolution and soil management legacy on the maize rhizobiome. Soil Biol. Biochem. 145:107794.

Shapiro, C. A., Ferguson, R. B., Hergert, G. W., Wortmann, C. S., and Walters, D. T. 2008. Fertilizer Suggestions for Corn. University of Nebraska-Lincoln Extension, Lincoln, NB, U.S.A.

Silva, U. C., Medeiros, J. D., Leite, L. R., Morais, D. K., Cuadros-Orellana, S., Oliveira, C. A., Lana, U. G. D., Gomes, E. A., and Dos Santos, V. L. 2017. Long-term rock phosphate fertilization impacts the microbial communities of maize rhizosphere. Front. Microbiol. 8:1266.

Sridevi, M., and Mallaiah, K. V. 2009. Phosphate solubilization by Rhizobium strains. Indian J. Microbiol. 49:98-102.

Szoboszlay, M., Lambers, J., Chappell, J., Kupper, J. V., Moe, L. A., and McNear, D. H. 2015. Comparison of root system architecture and rhizosphere microbial communities of Balsas teosinte and domesticated corn cultivars. Soil Biol. Biochem. 80:34-44.

Thomma, B. 2003. Alternaria spp.: From general saprophyte to specific parasite. Mol. Plant Pathol. 4:225-236.

Trivedi, P., Leach, J. E., Tringe, S. G., Sa, T. M., and Singh, B. K. 2020. Plant-microbiome interactions: From community assembly to plant health. Nat. Rev. Microbiol. 18:607-621.

USDA-NASS. 2021. Objective Yield Survey 1990-2021. United States Department of Agriculture National Agricultural Statistics Service. https:// www.nass.usda.gov/Surveys/Guide_to_NASS_Surveys/Objective_Yield/ index.php

Walters, W. A., Jin, Z., Youngblut, N., Wallace, J. G., Sutter, J., Zhang, W., Gonzalez-Pena, A., Peiffer, J., Koren, O., Shi, Q. J., Knight, R., del Rio, T. G., Tringe, S. G., Buckler, E. S., Dangl, J. L., and Ley, R. E. 2018. Large-scale replicated field study of maize rhizosphere identifies heritable microbes. Proc. Natl. Acad. Sci. U.S.A. 115:7368-7373.

White, T. J., Bruns, T., Lee, S., and Taylor, J. 1990. Amplification and direct sequencing of fungal ribosomal RNA genes for phylogenetics. Pages 315322 in: PCR Protocols: A Guide to Methods and Applications. M. A. Innis, D. H. Gelfand, J. J. Sninsky, and T. J. White, eds. Academic Press, San Diego, CA, U.S.A

Yao, Y. S., Sun, T., Wang, T., Ruebel, O., Northen, T., and Bowen, B. P. 2015. Analysis of metabolomics datasets with high-performance computing and metabolite atlases. Metabolites 5:431-442.

Zeffa, D. M., Perini, L. J., Silva, M. B., de Sousa, N. V., Scapim, C. A., de Oliveira, A. L., do Amaral, A. T., and Goncalves, L. S. A. 2019. Azospirillum brasilense promotes increases in growth and nitrogen use efficiency of maize genotypes. PLoS One 14:e0215332.

Zhalnina, K., Louie, K. B., Hao, Z., Mansoori, N., da Rocha, U. N., Shi, S. J., Cho, H. J., Karaoz, U., Loque, D., Bowen, B. P., Firestone, M. K., Northen, T. R., and Brodie, E. L. 2018. Dynamic root exudate chemistry and microbial substrate preferences drive patterns in rhizosphere microbial community assembly. Nat. Microbiol. 3:470-480.

Zheng, B. X., Bi, Q. F., Hao, X. L., Zhou, G. W., and Yang, X. R. 2017. Massilia phosphatilytica sp. nov., a phosphate solubilizing bacteria isolated from a long-term fertilized soil. Int. J. Syst. Evol. Microbiol. 67: 2514-2519. 